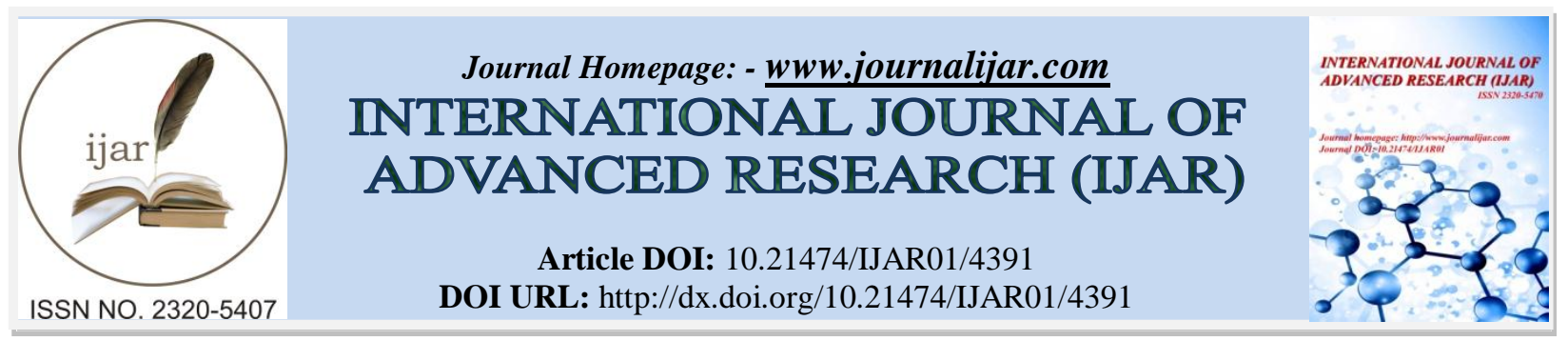

RESEARCH ARTICLE

\title{
DIAGNOSIS AND THERAPEUTIC MANAGEMENT OF CANINE EHRLICHIOSIS.
}

\author{
S. Ayodhya. \\ Associate Professor, Department Veterinary Medicine, College of Veterinary science, PVNR Telangana Veterinary \\ University, Korutla, 505326.
}

\section{Manuscript Info}

Manuscript History

Received: 04 april 2017

Final Accepted: 06 june 2017

Published: june 2017

Key words:-

Dogs, Snap 4dx test, Doxycycline.

\section{Abstract}

Two dogs were presented with history of canine erhlichiosis to Teaching Veterinary Clinical Comples, C.V.Sc, Korutla, Telangana. Two dogs were come with complaint of pale yellow conjuctival and buccal mucous membrane, high temperature, gradual dyspepsia from one month, passing yellow urine, discharge from the nose and eyes, edema of the legs, scrotum, vocational vomitions and patchy lesions on the body. Serological reports (Snap 4DX test) showed that positive for Ehrlichiosis organism. The oxytetracycline injection was continued for 4 day until stoppage of vomitions and fallowed by doxycycline @ 10mg/kg/body weight orally for 3 weeks along with liver tonic. After 24 days of treatment both dogs were become normal and active.

Copy Right, IJAR, 2017,. All rights reserved.

\section{Introduction:-}

Canine ehrlichiosis is a tick-borne disease of canines caused by the obligate intracellular organism, Ehrlichia canis. The arthropod vector of E. canis is Rhipicephalus sanguineus (Aguiar et al., 2007). In addition to its global importance to canine health, this vector-pathogen vertebrate interaction provides useful transmission and disease models for zoonotic tick-borne bacteria, the majority of which naturally infect dogs (Dumler et al.,2001). The parasite enters the blood stream and lymphatics and localizes in the macrophages of the reticuloendothelial system in the spleen, liver and lymph nodes, where it replicates by binary fission(Lakkawar et al., 2003). Ehrlichiosis is a serious febrile and potentially fatal disease of dogs usually diagnosed by clinical signs, although non-significant and pathognomic signs are present (Woody and Hoskins, 1991). The tetracyclines both oral and parenteral are the drugs of choice and are effective treatment of $E$. canis (Davidson et al., 1998). Keeping the significance of diagnosis and management of ehrlichiosis in dogs the present study was designed and reported.

\section{Materials And Methods:-}

Dogs of German shepherd and non descript were presented with the signs of recurrent fever, off food, edema of dependent parts and epistaxis to Teaching Veterinary Clinical Complex of CVSc, Korutla were considered for the study. Whole blood was collected for hematology and serum chemistry. Further, the suspected dogs were subjected for SNAP 4DX plus test (IDEXX Laboratories, United States) for confirmation of canine ehrlichiasis. Later the dogs that were confirmed for E. canis were subjected for treatment with injection oxytetracycline $10 \mathrm{mg} / \mathrm{kg}$ body weigt for 4 days until control of vomitions fallowed by oral doxycycline $10 \mathrm{mg} / \mathrm{kg}$, for 3 weeks. 


\section{Case Study And Clinical Examination:-}

Two dogs were presented with history of canine erhlichiosis to Teaching Veterinary Clinical Comples, C.V.Sc, Korutla, Telangana. One German shepherd dog was come with complaint of pale yellow conjuctival and buccal mucous membrane, temperature was recorded $105^{\circ} \mathrm{F}$, gradual dyspepsia from one month, passing yellow urine and sometimes it was have vomitions. Secondly non descript dog, was come with history of discharge from the nose and eyes and edema of the legs and scrotum, dyspepsia, vocational vomitions and patchy lesions on the body with high temperature $\left(106^{\circ} \mathrm{F}\right)$. Both dogs were treated in different hospitals with ceftriaxone injection along with antipyretic melaxicum. But both dogs were not responded to this treatment.

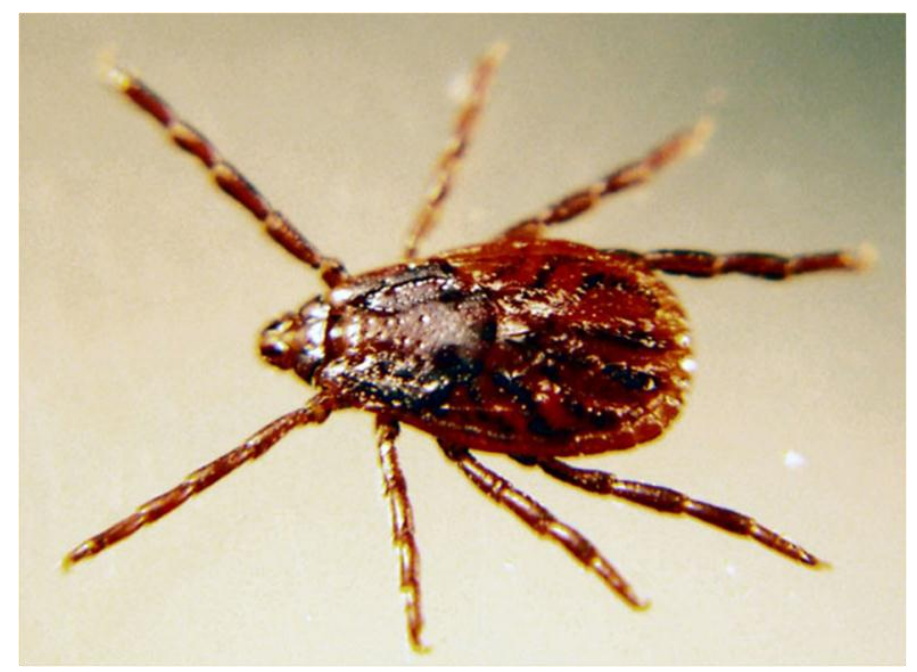

Rhipicephalus sanguineus tick.

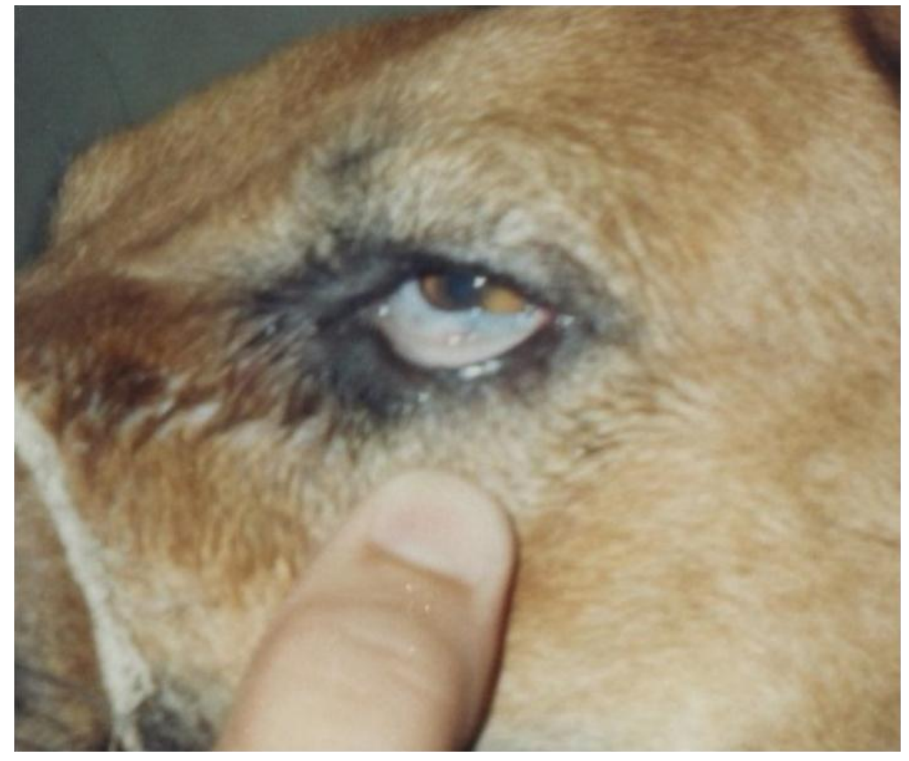

Icteric conjunctival mucous membranes. 


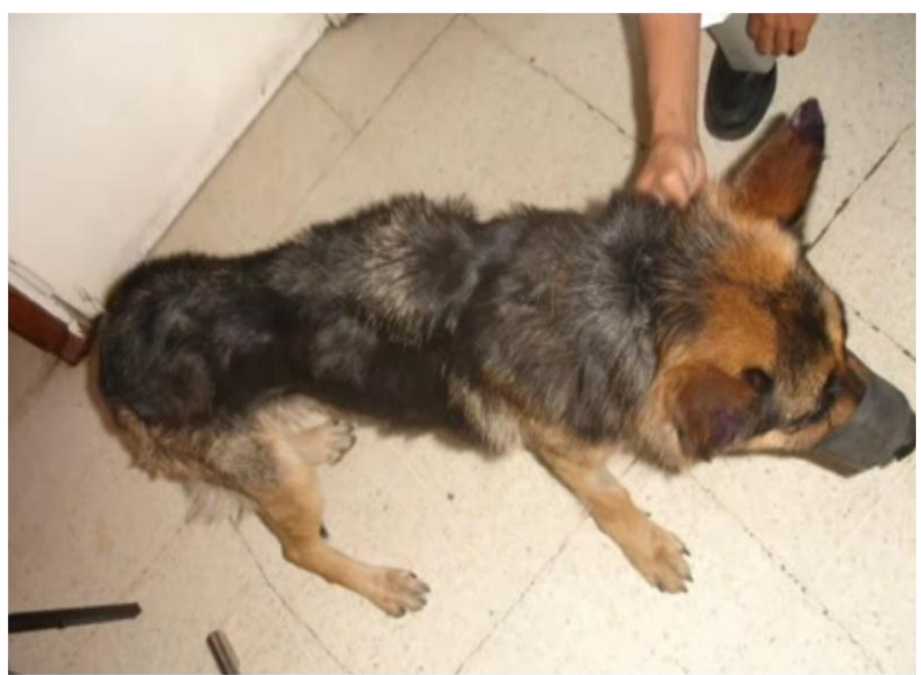

Unable to tolerate body weight.

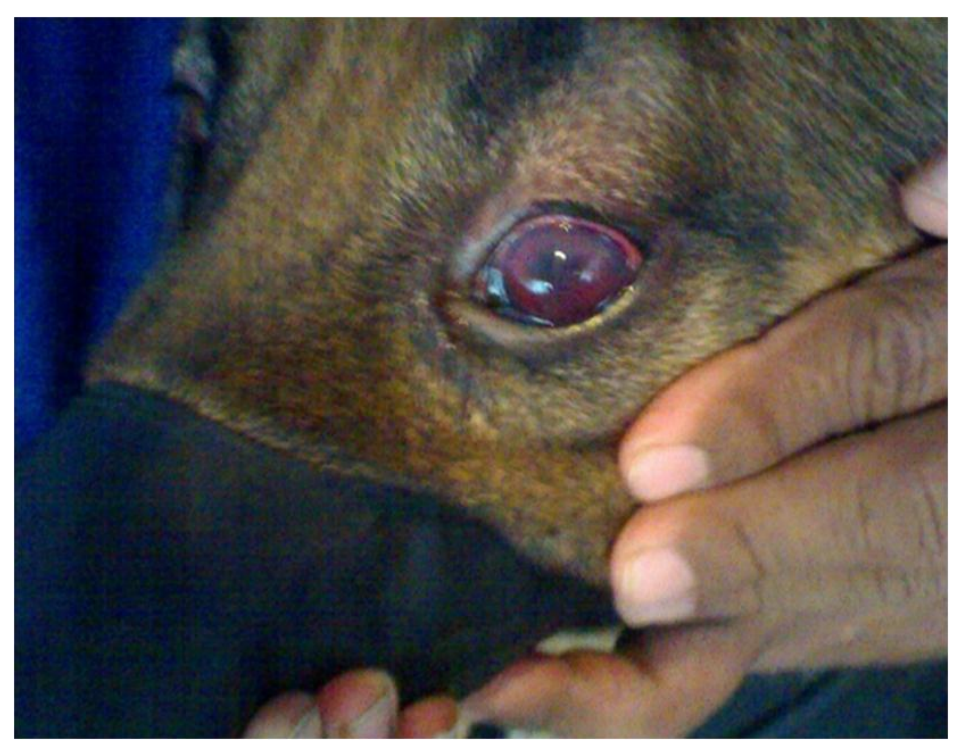

Hemorrhages in eye ball. 


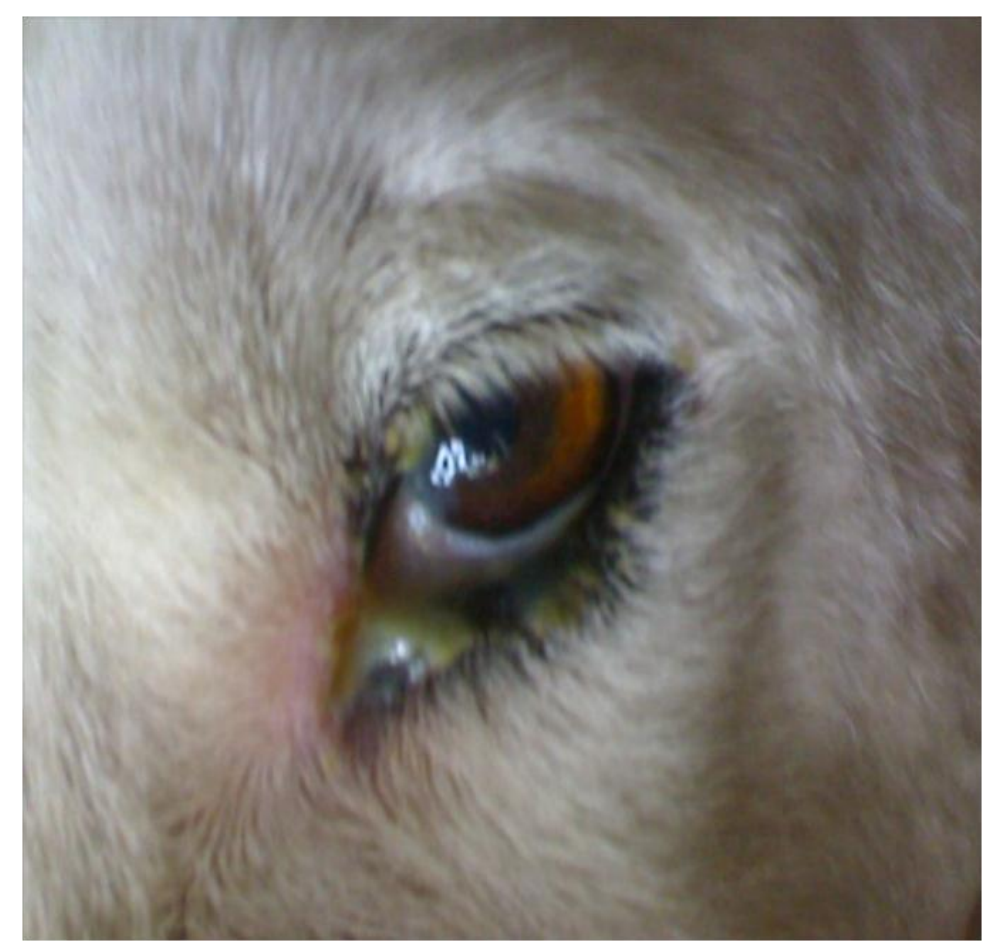

Ocular discharges.

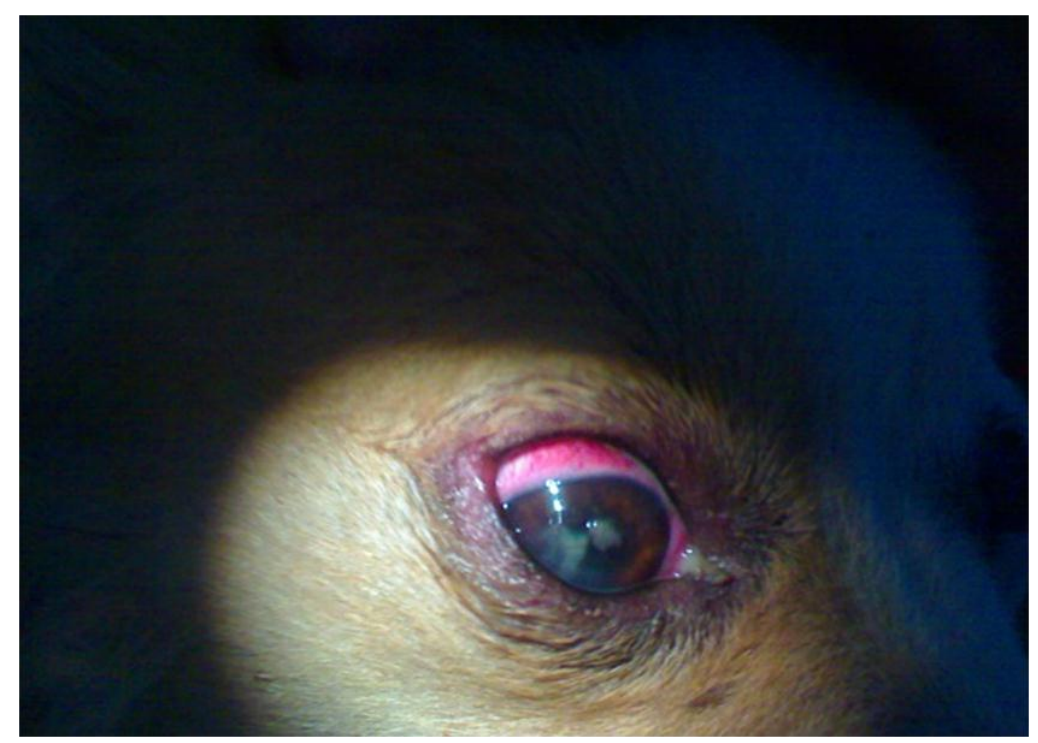

Congestion of CMM. 


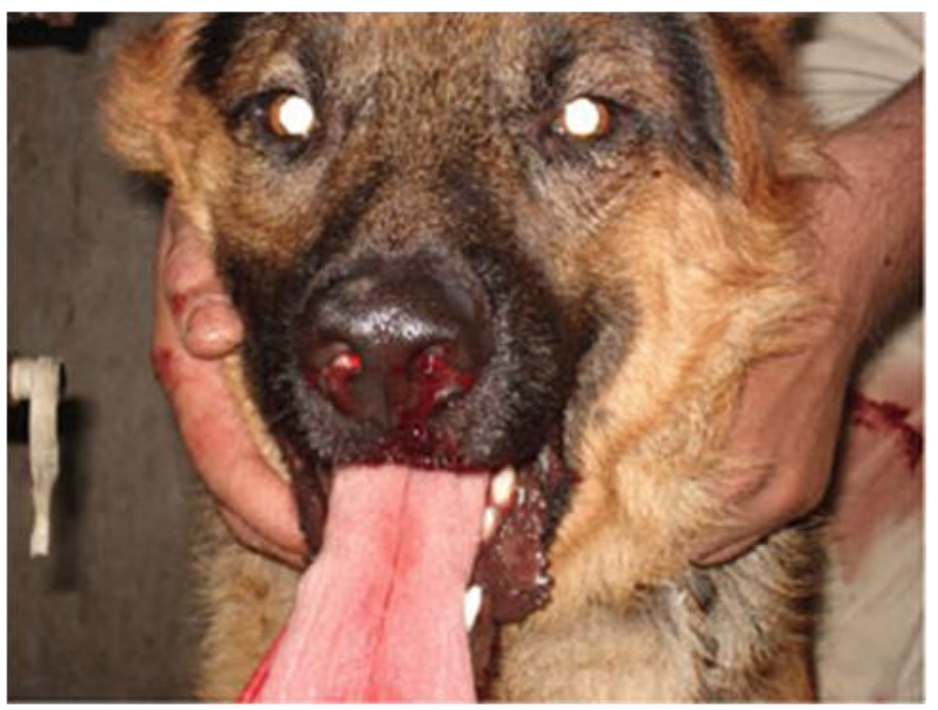

Epistaxis.

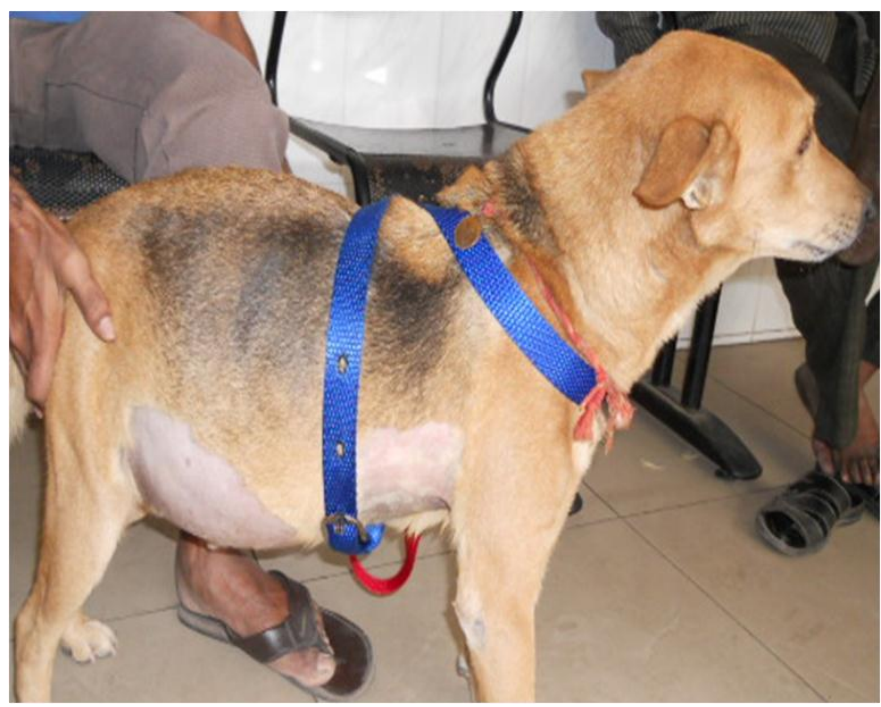

Ascites.

\section{Diagnosis and Therapy:-}

With the history of clinical signs tentatively diagnosed as ehrlichiosis. Blood sample was collected for confirmative diagnosis of ehrlichiosis. On the basis of examination of thin blood smear stained with Giemsa stain, all two cases were found to be positive for morula stage of Ehrlichia canis in monocytes. Serological reports (Snap 4DX test) showed that positive for Ehrlichiosis organism in both dogs. After tentative diagnosis therapy was given with injection of oxytetracycline at @ $10 \mathrm{mg} / \mathrm{kgbwt}$ intravenously along with fluid therapy and antipyretic drug melaxicam $0.2 \mathrm{mg} / \mathrm{kg}$ body weight subcutaneously and continued for 4 days until to control of vomitions. and fallowed by doxycycline was given @ 10mg/kg/body weight orally for 3 weeks along with liver tonic. 


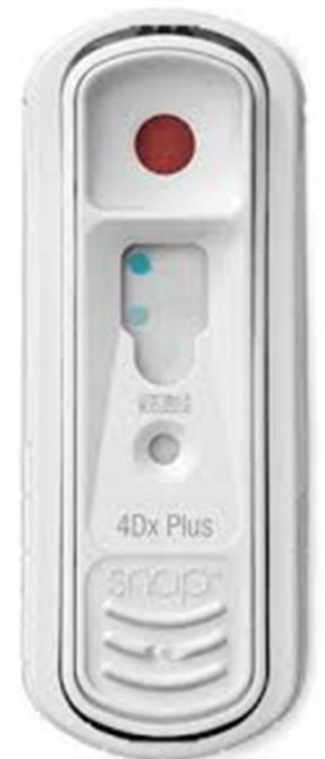

IDEXX Snap 4Dx plus test.

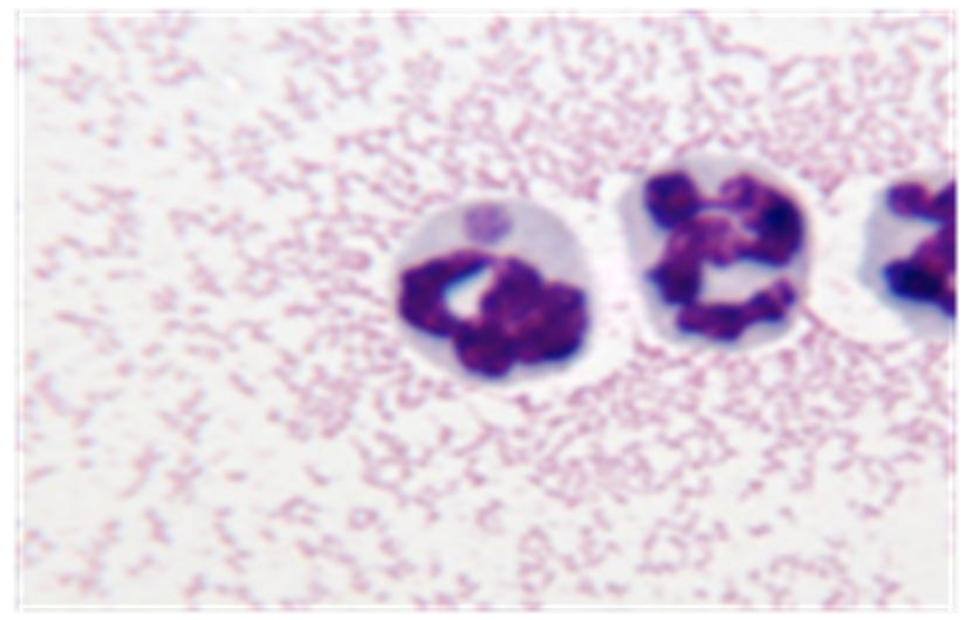

10. E.canis morula in monocytes

\section{Results and Discussion:-}

Canine eherlichiosis is acute, subclinical or chronic disease which is manifested as multi systemic forms. E. canis can infect all breeds of dogs but the German shepherd breed appears to be more susceptible, showing the more severe form of the disease with a higher morbidity and mortality compared to other breeds (Nyindo et al., 1980). In present study a thin smear was prepared for confirmation of ehrlichiosis stained with Gimesa stain and examined microscopically for presence of morula in monocytes and blood sample reports shows that decreased amount of platelets $40,000 \mathrm{~m} / \mathrm{l}$ in first dog and 55,000/ml in second dog. The findings of present study were similar to Waner et al., (1995) and (Emek Tuna and Ulutas, 2009) who reported, significant thrombocytopenia develops in experimentally infected dogs in approximately 1 week post-infection. Serological reports (Snap 4DX test) showed that positive for Ehrlichiosis organism in both dogs. Stillman and Monn (2014) documented that the SNAP 4Dx Plus Test, detects antibodies produced by five pathogens, which include A. phagocytophilum, A.platys, B. burgdorferi, E. canis and E. ewingii, in addition to detection of heartworm antigen. Further, Thatcher et al.,(2015) also opined that the IDEXX SNAP 4Dx Plus Test was substantially more sensitive than other rapid in-clinic serologic assays for detection of antibodies to canine monocytic ehrlichiosis. The dogs were responded slowly to the treatment by showing improvement in appetite and recorded decreased temperature. These findings are similar to the Harrus et al., (2004) reported that most dogs recover from acute and subclinical disease when treated with appropriate and adequate dosages of doxycycline or other tetracyclines which are the first line of drugs for ehrlichiosis. After 
therapeutic regimen followed, dogs showed remarkable improvement in blood picture, platelets count and negative reports for ehrlichiosis.

\section{Conclusion:-}

The antibiotics, tetracycline and doxycycline were used. The animals showed substantial improvement in condition after 2-3 days, started taking food. Oxytetracycline and Doxycycline both are the drug of choice for canine ehrlichiosis. The clinical recovery can be observed within 2-3 day of therapy and regimen needs to be continued for 3-4 weeks for clearing up parasitemia.

\section{Acknowledgement:-}

The authors are thankful to the Teaching Veterinary Clinical Comples, C.V.Sc, Korutla, Telangana, India for providing necessary facilities to carry out this work.

\section{Competing Interests:-}

The authors declare that they have no competing interests.

\section{References:-}

1. Aguiar, D.M., Cavalcante, G.T., Pinter, A., Gennari, S.M., Camargo, L.M. and Labruna, M.B. 2007: Prevalence of Ehrlichia canis (Rickettsiales:Anaplasmataceae) in dogs and Rhipicephalus sanguineus (Acari: Ixodidae) ticks from Brazil. J Med Entomol., 44 (1), 126-132.

2. Davidson, D.E., Dill, G.S., Tingpalapong, M., Premabutra, S., Nguen, P.L., Stephenson, E.H. and Ristic, M. 1998: Prophylactic and therapeutic use of tetracycline during anepizootic of ehrlichiosis among military dogs. $J$. Am. Vet.Med. Assoc., 172:697-700.

3. Dumler, J.S., Barbet, A.F., Bekker, C.P., Dasch, G., Palmer, A.G.H., Ray, S.C., Rikihisa, Y. and Rurangirwa, F.R.. 2001: Reorganization of genera in the families Rickettsiaceae and Anaplasmataceae in the order Rickettsiales: unification of some species of Ehrlichia with Anaplasma, Cowdria with Ehrlichia and Ehrlichia with Neorickettsia,descriptions of six new species combinations and designation of Ehrlichia equi and 'HGE agent' as subjective synonyms of Ehrlichia phagocytophila. Int. J. Syst. Evol. Microbiol., 51:2145-2165.

4. Emek Tuna, G. and Ulutas, B. 2009: Prevalence of ehrlichia canis infection in thrombocytopenic dogs .Lucrări stiinłifice MEDICINĂ VETERINARĂ VOL. XLII, University of Adnan Menderes, Faculty of Veterinary Medicine, Department of Internal Medicine, 09016 AYDIN/TURKEY.

5. Nyindo, M., Huxsoll, D.L., Ristic, M., Kakoma, I., Brown, J.L., Carson, C.A. and Stephenson, E.H. 1980: Cellmediated and humoral immune responses of German Shepherd Dogs and Beagles to experimental infection with Ehrlichia canis. Am. J. Vet. Res., 41(2): 250-254.

6. Waner, T., Harrus, S., Bark, H., Bogin, E., Avidar, Y. and Keysary, A. 1997: Characterization of the subclinical phase of canine ehrlichiosis in experimentally infected beagle dogs. Vet. Parasitol., 69(3): 307-317.

7. Waner, T., Harrus, S., Jongejan, F., Bark, H., Keysary, A. and Cornelissen, A.W. 2001: Significance of serological testing for ehrlichial diseases in dogs with special emphasis on the diagnosis of canine monocytic ehrlichiosis caused by Ehrlichia canis. Vet. Parasitol., 95(1): 1-15.

8. Stillman, B. A., Monn. M. and Liu, J. 2014: Performance of a commercially available in-clinic ELISA for detection of antibodies against Anaplasma phagocytophilum, Anaplasma platys, Borrelia burgdorferi, Ehrlichia canis, and Ehrlichia ewingii and Dirofilaria immitis antigen in dogs. J. Am. Vet. Med. Assoc., 245(1):80-86.

9. Thatcher, B., Liu, J., Andrews, B., Breitschwerdt, E., Beall, M. and Chandrashekar, R. 2015: Comparative evaluation of two rapid in-clinic serologic assays for detection of antibodies to canine monocytic and granulocytic ehrlichiosis. Presented at the 27th Meeting of the American Society for Rickettsiology; June 2023, 2015

10. Procajlo, A., Mikulska Skupiei, E., Bladowski, M. and Lew, S. 2011: Monocytic Ehrlichiosis in dogs. Polish Journal of Veterinary Sciences. 14( 3): 515-520

11. Lakkawar, A.W., Nair, M.G., Varshney, K.C., Sreekrishnan, R., Rao, V.N. 2003: Pathology of canine monocytic ehrlichiosis in a german shepherd dog. Slov Vet Res., 40 (2): 119-128 